\title{
INAPPROPRIATE S-ICD PATIENT RECEIVES FALSE POSITIVE SHOCKS
}

\section{David Korpas}

\author{
Institute of Nursing, Faculty of Public Policies, Silesian University, Opava, Czech Republic
}

\begin{abstract}
Subcutaneous implantable cardioverter defibrillator (S-ICD) protects the patients at risk for sudden cardiac death while leaving the heart and vasculature untouched. It provides life-saving therapy, but may also deliver inappropriate therapy. Presented case demonstrates a possibility of S-ICD therapy induction due to double-counting. It was original caused by lack of suitable sensing vectors and the solution was possible just particularly. As the inter-individual variability of subcutaneous cardiac signal is considerable, the patient screening should be necessary for identification of such patients, which have an unsuitable subcutaneous sensing signals.
\end{abstract}

\section{Keywords}

false-positive therapy, defibrillation, implantable cardioverter-defibrillator, patient screening

\section{Introduction}

The S-ICD system provides defibrillation therapy from outside of the heart. It introduces completely subcutaneous solution for patients at risk for sudden cardiac arrest after removal of conventional ICD, or for young patient without need of brady pacing. At the implant, there is no risk of vascular injury, low risk of systemic infection and no intra-cardiac biomechanical stresses. It does not require any leads in the heart and leave it untouched [1]. The system consists of a subcutaneous device and a lead, placed along the sternum approximately $1 \mathrm{~cm}$ on the left side, see Fig. 1 . Since the system is placed by anatomical landmarks, the implant also does not require the fluoroscopy [2].

The S-ICD device algorithms should provide performance comparable to transvenous ICDs [3]. The detection is based not only on heart rate criteria, but also on morphological criteria. As the defibrillation is provided from longer distance, the delivered shock energy is higher, as of 80 joules, within $\leq 10$ seconds of charge time.

The lead is implanted subcutaneously, with the distal portion positioned parallel to the left sternal border and the proximal end of the lead connected to the S-ICD device. The lead is just $45 \mathrm{~cm}$ length, with distal sensing surface area of $46 \mathrm{~mm}^{2}$, proximal sensing surface area of $36 \mathrm{~mm}^{2}$. Distal electrode location is at the tip, proximal electrode is $120 \mathrm{~mm}$ from tip. Defibrillation surface area is $750 \mathrm{~mm}^{2}$. The materials used are same as for transvenous defibrillation lead. The lead is designed to withstand cardiopulmonary resuscitation forces.

The programming system is battery operated, enables wand or RF telemetry and wireless printing. The tachycardia detection is accomplished by one out of three far-field sensing vectors, called primary, secondary and alternate (see Fig. 1). These can be automatically set-up or manual selected. It enables certain flexibility to solve sensing issues non-invasively and some of the clinical events can be managed through sensing vector changes. The entry ECG screening initial and selection of sensing vector are therefore of critical importance.

The screening ECG is a modified three-channel surface ECG, which mimics the sensing vectors of the S-ICD system [4]. Placement of surface electrodes provides vectors similar to the sensing configuration used by the device and the surface ECG is representative of the subcutaneous signal. The screening process is used to analyze QRS amplitude, QRS to T-wave amplitude ratios, QRS width and morphology consistency in sinus rhythm using multiple patient postures - standing, sitting and supine position.

For the screening, it is important to establish a stable surface ECG baseline. If not available, the ground electrode can be added, the skin preparation can be improved or new electrodes can be used. Typically, the surface ECG electrodes should be positioned as follows:

- $\quad$ Electrode LL: placed in a lateral location, at the 5th intercostal space along the mid-axillary line to rep- 
resent the intended location of the implanted pulse generator;

- Electrode LA: placed $1 \mathrm{~cm}$ left lateral of the xiphoid midline to represent the intended location of the proximal sensing node of the implanted electrode;

- $\quad$ Electrode RA: placed $14 \mathrm{~cm}$ superior to the ECG Electrode LA, to represent the intended position of the distal sensing tip of the implanted electrode [4].

The surface ECG is recorded in these location that represents the intended position of the implanted S-ICD. If a non-standard S-ICD electrode or pulse generator placement is desired, the surface ECG electrode locations should be modified accordingly.

Records of 10-20 seconds of ECG should be acquired in at least two different patient postures (supine and standing, supine and sitting), with the following ECG settings: leads I, II and III, sweep speed of $25 \mathrm{~mm} / \mathrm{sec}$, ECG gain 5-20 mm/mV.

Other postures may include: left lateral, right lateral, and prone. If morphology changes are expected at elevated heart rates, screening ECGs can be collected during exercise testing for evaluation purposes. If the S-ICD is to be implanted with a concomitant pacemaker, all ventricular morphologies (paced and intrinsic, if normal conduction is expected) should be collected [2].

The evaluation of each QRS complex is a three-steps process:

- select the profile,

- align the profile,

- $\quad$ evaluate the QRS complex.

As a profile reference, the transparent screening ruler serves. First select the profile from the ruler, which best matches the amplitude of the QRS complex. The peak of the QRS complex must fit within one of the peak zones. For biphasic signals, the larger peak should be used to select the profile. Align the left edge of the selected profile with the onset of the QRS complex. The horizontal line on the profile should be used as a guide for the ECG baseline. Then evaluate each QRS complex. For acceptable complex the entire QRS complex and trailing T-wave are contained within the profile. For unacceptable complex some portion of the QRS complex or trailing T-wave extends outside of the profile. The evaluation process is repeated for all QRS complexes from all surface ECG leads in all collected postures [5].

This can be also done automatically using the software screening tool in the common manufacturer's programmer. A patient is considered suitable for implant if at least one surface ECG lead (sensing vector) is acceptable for all tested postures. Unacceptable or marginal sensing vectors may result in inappropriate or failure to deliver therapy [2].
After the implant, the device provides the sensing and detection process by utilizing three simultaneous rhythm analyses:

- static morphology analysis identifies non-shockable rhythms, utilizing the normal sinus rhythm (NSR) template;

- dynamic morphology analysis identifies shockable polymorphic rhythms by comparing each complex to the previous ones;

- QRS width analysis compares the QRS width to the NSR QRS width.

Generally, the detection algorithm identifies and evaluates overall heart rhythm, rather than the individual beats as it is in comment defibrillation systems.

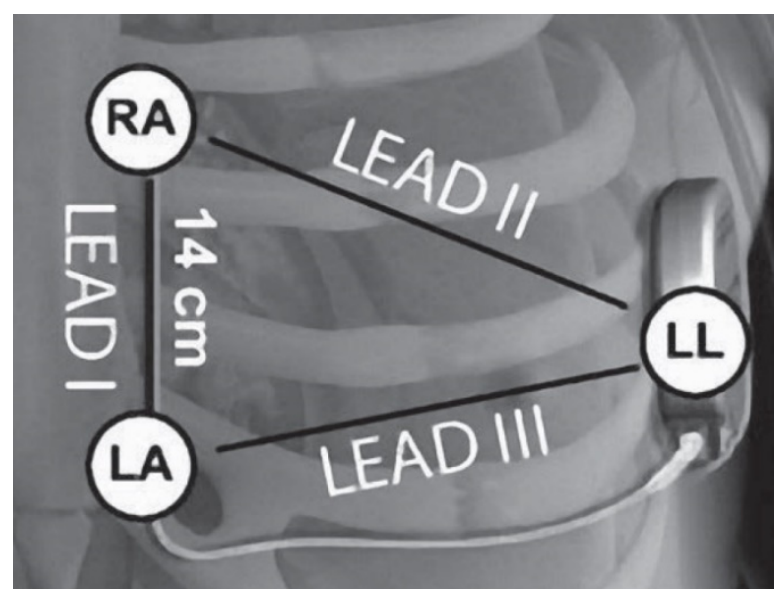

Fig. 1: The placing of S-ICD system and sensing vectors: primary (Lead III), secondary (Lead II) and alternate (Lead I). The ECG lead wire colors corresponds to the traffic light colors: $R A=$ red, $L A=$ yellow, $L L=$ green [6].

\section{Methods}

The case of inappropriate shock was reported by the patient twenty-two days after the implant. He performed sport. The device was programmed according to current clinical recommendations. Conditional tachycardia zone was set to $200 \mathrm{~min}^{-1}$ and shock zone to $220 \mathrm{~min}^{-1}$ and primary sensing configuration (Fig. 2).

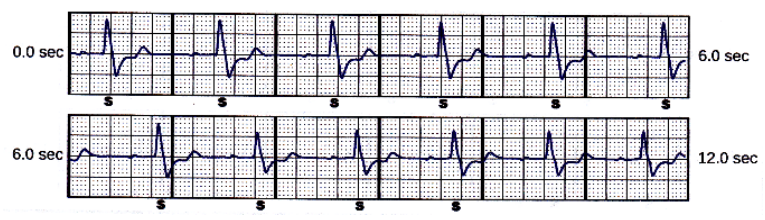

Fig. 2: Primary sensing vector snapshot. 


\section{Case report}

A seventy-one-old, physically very active man patient with history of perioperative ventricular tachycardia was indicated to primary prevention defibrillation implant. First, he received a conventional single chamber ICD several years ago. During this time, the patient did not receive any therapy. Because of mechanical damage of the lead, the system was explanted and the patient has received the subcutaneous implantable cardioverter defibrillator.

Twenty-two days after the implant, the patient came to the ambulatory, complaining to false-positive shock. As the S-ICDs provide the shocks with higher energy of up to $80 \mathrm{~J}$, it is more painful than shocks from the conventional ICD.

The device memory contained one saved treated episode. It is shown in Fig. 3. The episode was caused be double counting, i.e. T-wave oversensing.

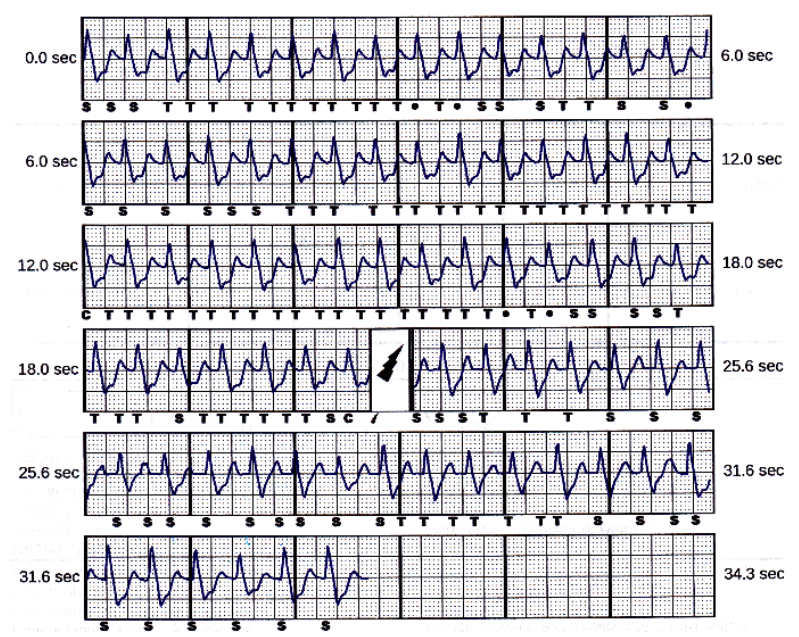

Fig. 3: Treated episode saved in the device memory. Beats marked "S" are sensed (below the tachycardia detection zone), beats marked " $T$ " are in tachycardia zone, " $C$ " means charge started. The symbol of flash stands for the delivered shock.

The delivered $80 \mathrm{~J}$ shock had no impact on the rhythm. The episode was terminated after spontaneous decrease of patient's heart rate below $110 \mathrm{~min}^{-1}$. The programmed sensing configuration was therefore obviously not optimal.

However, the next two sensing vectors (secondary and alternate) have also high T-wave, see Fig. 4 and Fig. 5.

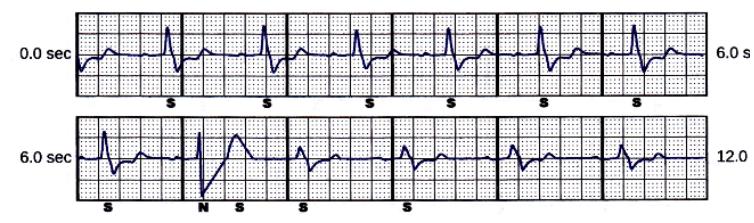

Fig. 4: Secondary sensing vector snapshot. Beat marked " $N$ " is noise.

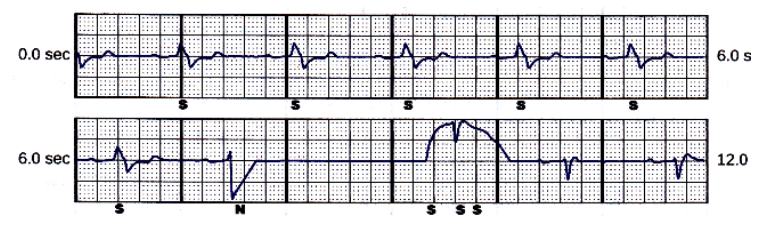

Fig. 5: Alternate sensing vector snapshot. Beat marked " $N$ " is noise.

The device can automatically choose the best sensing vector. Therefore, the chance to improve the situation was limited. The tachycardia detection zones were moved to $220 \mathrm{~min}^{-1}$ (conditional tachycardia zone) and $240 \mathrm{~min}^{-1}$ (shock zone). The sensing configuration was changed to secondary, as the T-wave amplitude looked smaller, respectively the $\mathrm{R} / \mathrm{T}$ wave ration was better comparing to alternate vector. The patient was instructed not to exceed the heart rate of $110 \mathrm{~min}^{-1}$ (half of conditional zone limit $220 \mathrm{~min}^{-1}$ ).

Unfortunately, the patient received a new inappropriate shock during the next day from the same reason (T-wave oversensing). As there are no other adjustable discrimination criteria except of heart rate, one zone only was programmed to $250 \mathrm{~min}^{-1}$, as the last possible solution. Taking the double-counting into account, the patient is now save just up to $125 \mathrm{~min}^{-1}$ of sinus heart rate.

\section{Conclusion}

This case report emphasizes the need for screening of the patient for the subcutaneous ECG vectors morphology before the S-ICD implant. The real sudden cardiac death risk of the patient should also be considered, especially in secondary prevention. The reported patient remains in difficult situation, his possibilities for sports or other physical activities are now very limited. It can be concluded, that he was an inappropriate candidate for S-ICD implant, because of no suitable sensing vector. 


\section{References}

[1] Weiss R, Knight BP, Gold MR, Leon AR, Herre JM, Hood M, et al. Safety and efficacy of a totally subcutaneous implantablecardioverter defibrillator. Circulation. 2013 Aug 27;128(9): 944-53. DOI: 10.1161/CIRCULATIONAHA.113.003042

[2] Boston Scientific. User's manual EMBLEM ${ }^{\mathrm{TM}}$ S-ICD, EMBLEM $^{\mathrm{TM}}$ MRI S-ICD.

https://www.bostonscientific.com/content/dam/bostonscientifi c/Rhythm\%20Management/portfolio-group/EMBLEM_SICD/Download Center/359481-001\%20EMBLEM\%20SICD\%20PTM_English.pdf (22nd June 2019 date last accessed)

[3] Gold MR, Theuns DA, Knight BP, Sturdivant JL, Sanghera R, Ellenbogen KA, et al. Head-to-Head Comparison of Arrhythmia Discrimination Performance of Subcutaneous and Transvenous ICD Arrhythmia Detection Algorithms: The START Study. Journal of Cardiovascular Electrophysiology. 2011 Oct 28;23(4):359-66. DOI: 10.1111/j.1540-8167.2011.02199.x

[4] Bellardine Black CL, Stromberg K, Van Balen GP, Ghanem RN, Breedveld RW, Tieleman RG. Is surface ECG a useful surrogate for subcutaneous ECG? Pacing Clin Electrophysiol. 2010 Jan 24;33(2):135-45. DOI: 10.1111/j.1540-8159.2009.02616.X

[5] Zeb M, Curzen N, Veldtman G, Yue A, Roberts P, Wilson D, et al. Potential eligibility of congenital heart disease patients for subcutaneous implantable cardioverter-defibrillator based on surface electrocardiogram mapping. Europace. 2015 Feb 12; 17(7),1059-67. DOI: 10.1093/europace/euu375

[6] Ali H, Lupo P, Cappato R. The Entirely Subcutaneous Defibrillator - A New Generation and Future Expectations. Arrhythmia \& Electrophysiology Review. 2015 Aug;4(2):116-21. DOI: 10.15420/aer.2015.04.02.116

David Korpas, Ph.D. Institute of Nursing Faculty of Public Policies Silesian University in Opava Bezručovo náměstí 885/14, CZ-746 01 Opava

E-mail: david.korpas@seznam.cz Phone: +420 553684160 